\title{
Eficácia da N-Butilescopolamina e Dipirona Sódica Associadas ao Cetoprofeno no Alívio da Dor Pós-Operatória de Pacientes Submetidas a Duas Técnicas Diferentes de Laqueadura por Laparoscopia *
}

\section{Efficacy of N-Butylscopolamine and Sodium Dipirone Associated to Ketoprofen for Postoperative Pain Relief of Patients Submitted to Two Different Laparoscopic Sterilization Techniques}

Eliana Marisa Ganem, TSA ${ }^{1}$; Isabel C F Salem ${ }^{2}$; Fernanda B Fukushima ${ }^{3}$; Giane Nakamura ${ }^{4}$; Rogério Dias ${ }^{5}$; André L Fontana ${ }^{6}$; Nilton J Leite ${ }^{6}$

\section{RESUMO}

Ganem EM, Salem ICF, Fukushima FB, Nakamura G, Dias R, Fontana AL, Leite NJ - Eficácia da N-Butilescopolamina e Dipirona Sódica Associadas ao Cetoprofeno no Alívio da Dor Pós-Operatória de Pacientes Submetidas a Duas Técnicas Diferentes de Laqueadura por Laparoscopia

JUSTIFICATIVA E OBJETIVOS: A laqueadura laparoscópica (LL) é um dos procedimentos mais dolorosos e a intensidade da dor varia com a técnica selecionada, sendo mais intensa com a técnica de oclusão das tubas uterinas com anel. As pacientes submetidas à $L L$ referem dor em cólica no período PO e a N-butilescopolamina e a dipirona sódica, por suas propriedades anti-espasmódicas e analgésicas, associadas às propriedades antiinflamatórias do cetoprofeno, podem ser opção para profilaxia e tratamento de dor. O objetivo deste foi estudar a eficácia da $\mathrm{N}$-butilescopolamina e da dipirona sódica associadas ao cetoprofeno, na prevenção de dor $P O$ em pacientes submetidas à $L L$, com duas técnicas diferentes - diatermia e pinçamento com anel.

MÉTODO: Participaram do estudo 50 pacientes, estado físico ASA I e II, com idade entre 23 e 47 anos. As pacientes foram distribuidas aleatoriamente em dois grupos: G1 - oclusão das tubas uterinas com anéis, G2 - oclusão das tubas uterinas com diatermia. Todas as pacientes receberam $\mathrm{N}$-butilescopolamina $(20 \mathrm{mg})$ e dipirona sódica $(2500 \mathrm{mg})$ e cetoprofeno $(100 \mathrm{mg})$, por via venosa, imediatamente antes da indução da anestesia. A dor foi avaliada pelo critério de escala numérica verbal, variando de 0 a 10, sendo 0 ausência de dor e 10 o máximo de dor, a cada 10 minutos na primeira hora, na sala de recuperação pós-anestésica (SRPA) e na $1^{a}, 2^{a}, 3^{a}$ e $4^{a}$ horas após a alta da SRPA. Dor com intensidade maior que 3 era tratada com tramadol (100 mg), por via venosa. A avaliação da dor foi realizada sem que se soubesse a que grupo pertencia a paciente. Para análise estatística, testes $t$ de Student, Mann-Whitney e Friedman.

* Recebido do (Received from) Departamento de Anestesiologia da Faculdade de Medicina de Botucatu (FMB - UNESP), Botucatu, SP

1. Professora Adjunta do CET/SBA da FMB - UNESP

2. Aluna do $5^{\circ}$ ano de Graduação em Medicina, da Faculdade de Medicina de Botucatu - UNESP. Bolsa de Iniciação Científica PIBIC/CNPq

3. $\mathrm{ME}_{3}$ (2003) em Anestesiologia do CET/SBA da FMB - UNESP

4. Anestesiologista da FMB - UNESP

5. Professor Adjunto do Departamento de Ginecologia e Obstetrícia da FMB - UNESP

6. Médico do Departamento de Ginecologia e Obstetrícia da FMB - UNESP

Apresentado (Submitted) em 09 de setembro de 2004

Aceito (Accepted) para publicação em 13 de abril de 2005

Endereço para correspondência:

Profa. Dra. Adjunta Eliana Marisa Ganem

Faculdade de Medicina de Botucatu, UNESP

18618-970 Botucatu, SP

E-mail:eganem@fmb.unesp.br

(C) Sociedade Brasileira de Anestesiologia, 2005
RESULTADOS: Ambos os grupos foram idênticos com relação à idade, ao peso, à altura, à duração da cirurgia e anestesia. As pacientes do G1 apresentaram maior escore de dor que as do G2, em todos os momentos do estudo. Valores estatisticamente significativos: $80 \%$ das pacientes de G1 e $16 \%$ de $\mathrm{G} 2$ necessitaram de tramadol em algum momento do estudo.

CONCLUSÕES: A N-butilescopolamina e a dipirona sódica associadas ao cetoprofeno mostraram ser alternativa de analgesia pós-operatória quando a laqueadura é realizada com a técnica de diatermia.

Unitermos: ANALGÉSICOS, Antiinflamatório: cetoprofeno, dipirona; DOR, Aguda: pós-operatória; DROGAS: antimuscarínico

\section{SUMMARY}

Ganem EM, Salem ICF, Fukushima FB, Nakamura G, Dias R, Fontana AL, Leite NJ - Efficacy of N-Butylscopolamine and Sodium Dipirone Associated to Ketoprofen for Postoperative Pain Relief of Patients Submitted to Two Different Laparoscopic Sterilization Techniques

BACKGROUND AND OBJECTIVES: Laparoscopic sterilization (LS) is one of the most painful procedures and pain severity varies with the selected technique, being more severe when tubes are occluded with rings. Patients submitted to LS refer PO colic pain and N-butylscopolamine and sodium dipirone, for their anti-spasmodic and analgesic properties, associated to ketoprofen with anti-inflammatory properties, may be the option for pain prevention and relief. This study aimed at evaluating the efficacy of $\mathrm{N}$-butylscopolamine and sodium dipirone associated to ketoprofen to prevent $P O$ pain in patients submitted to $L S$ by two different techniques diathermy and tubal ring.

METHODS: Participated in this study 50 patients, physical status ASA I and II, aged 23 to 47 years. Patients were randomly distributed in two groups: G1 - tubal ring; G2 - diathermy. All patients received intravenous $\mathrm{N}$-butylscopolamine (20 mg) and sodium dipirone $(2500 \mathrm{mg}$ ) and ketoprofen $(100 \mathrm{mg})$ immediately before anesthetic induction. Pain was evaluated by verbal numeric scale varying from 0 to 10 , being 0 no pain and 10 the worst possible pain, every 10 minutes in the first PACU hour and 1, 2, 3 and 4 hours after PACU discharge. Pain above 3 was treated with intravenous tramadol (100 mg). Pain was evaluated without knowing the group to which the patient belonged. Student's $t$, Mann-Whitney and Friedman tests were used for statistical analysis.

RESULTS: Both groups were similar in age, weight, height and length of surgery and anesthesia. G1 patients had higher pain scores as compared to $\mathrm{G} 2$, in all studied moments. Statistically significant values were: $80 \%$ of G1 and $16 \%$ of $G 2$ patients needed tramadol at some point of the study.

CONCLUSIONS: N-butylscopolamine and sodium dipirone associated to ketoprofen are a postoperative analgesic alternative when sterilization is performed through diathermy technique.

Key Words: ANALGESICS, Anti-inflammatory: dipirone, ketoprofen; DRUGS: antimuscarinic; PAIN, Acute: postoperative 


\section{INTRODUÇÃO}

$D^{\alpha}$ or abdominal no pós-operatório (PO) é complicação comum em procedimentos laparoscópicos ${ }^{1-4}$. Há etiologia multifatorial colaborando para o seu aparecimento, como laceração de vasos sangüíneos, tração traumática de nervos, liberação de mediadores inflamatórios associada à rápida distensão peritoneal e estimulação do nervo frênico pelo gás carbônico residual na cavidade abdominal, levando a dor referida no ombro ${ }^{5}$.

A laqueadura por laparoscopia é um dos procedimentos laparoscópicos ginecológicos mais dolorosos. A intensidade da dor PO varia com a técnica escolhida para sua realização. O tratamento da dor $\mathrm{PO}$ após laqueadura pode ser realizado com a instilação de anestésico local nas tubas uterinas e no mesosalpinge, com o uso de antiinflamatórios não-esteroidais e com analgésicos opióides ${ }^{6-11}$. Os antiinflamatórios não foram suficientes para abolir a dor quando utilizados como agentes únicos ${ }^{12}$.

As pacientes submetidas à laqueadura referem dor em cólica no período $\mathrm{PO}$ imediato e a $\mathrm{N}$ - butilescopolamina e a dipirona sódica (Buscopan Composto ${ }^{\circledR}$ ), por suas propriedades anti-espasmódicas e analgésicas, associadas ao cetoprofeno, poderiam ser opção para profilaxia da dor. Contudo, estudos contestaram a eficácia da N-butilescopolamina no seu tratamento após a ligadura das tubas uterinas com pinças de Filshie ${ }^{13,14}$.

Como não existe na literatura pesquisa que utilize a N-butilescopolamina - dipirona sódica e cetoprofeno no controle da dor PO de laqueadura laparoscópica, estudou-se a eficácia desta associação em pacientes submetidas à esterilização com duas técnicas cirúrgicas diferentes, ou seja, diatermia e pinçamento com anéis.

\section{MÉTODO}

Após a aprovação do Comitê de Ética em Pesquisa Clínica e o consentimento por escrito, participaram do estudo 50 pacientes do sexo feminino, com idade entre 23 e 47 anos, estado físico ASA I e II, índice de massa corpórea inferior a 30, que foram submetidas à laparoscopia ginecológica para laqueadura das tubas uterinas. Foram excluídas do estudo aquelas que, no período pré-operatório, utilizaram drogas antiinflamatórias ou analgésicas, usuárias de álcool e drogas e estavam menstruadas. As pacientes foram distribuídas, por sorteio, em dois grupos de estudo que se diferenciaram pela técnica cirúrgica utilizada, ou seja, grupo 1, oclusão das tubas uterinas com anéis, e grupo 2 , oclusão das tubas uterinas com diatermia. Todas as pacientes receberam Nbutilescopolamina (20 mg) - dipirona sódica (2500 mg) e cetoprofeno (100 mg), por via venosa, imediatamente antes da indução da anestesia.

Após jejum de oito horas, as pacientes foram medicadas com $7,5 \mathrm{mg}$ de midazolam, por via oral, 60 minutos antes de serem encaminhadas ao centro cirúrgico. $\mathrm{Na}$ sala de operação, após obtenção do acesso venoso, foi instalada infusão de solução de Ringer com lactato $\left(10 \mathrm{~mL} \cdot \mathrm{kg}^{-1}\right)$ e administrado ondansetron (4 mg), para profilaxia de náusea e vômito.

Foi empregada monitorização com eletrocardioscópio na derivação $D_{\| l}$, esfigmomanômetro e oxímetro de pulso. Iniciou-se a indução anestésica com sufentanil $\left(0,5 \mu \mathrm{g} \cdot \mathrm{kg}^{-1}\right)$ e propofol $\left(2 \mathrm{mg} \cdot \mathrm{kg}^{-1}\right)$. Para auxiliar a intubação traqueal, foi administrado atracúrio $\left(0,5 \mathrm{mg} \cdot \mathrm{kg}^{-1}\right)$. Após a ventilação manual com oxigênio $\left(\mathrm{O}_{2}\right)$ a $100 \%$, realizou-se a intubação traqueal, a instalação da capnografia e a passagem de sonda orogástrica para escoamento do ar acumulado no estômago durante a ventilação manual, iniciando-se a administração de isoflurano. Utilizaram-se sistema de anestesia com reinalação e óxido nitroso $\left(\mathrm{N}_{2} \mathrm{O}\right)$ em fração inspirada de oxigênio de 0,4 . O volume corrente foi de $10 \mathrm{~mL} \cdot \mathrm{kg}^{-1}$ e a freqüência respiratória, suficiente para manter a pressão expirada de dióxido de carbono $\left(\mathrm{P}_{\mathrm{ET}} \mathrm{CO}_{2}\right)$, em torno de $30 \mathrm{mmHg}$.

Concluída a anestesia, após a reversão do bloqueio neuromuscular com atropina (1 mg) e neostigmina (1,5 mg), por via venosa, efetuava-se a extubação assim que as pacientes recuperavam ventilação espontânea eficiente e a consciência, quando, então, eram encaminhadas à SRPA. Na SRPA, as pacientes eram inquiridas com relação à dor, a cada 10 minutos durante uma hora. A dor foi avaliada pelo critério de escala numérica verbal (ENV), variando de 0 a 10 , sendo zero ausência de dore 10, a dor máxima. Se a intensidade da dor fosse superior a 3, administrava-se tramadol (50 mg) por via venosa. Se a dor persistisse, repetia-se o tramadol (50 mg). Se a dor ainda persistisse, seria administrada morfina por via venosa.

Na enfermaria, 1, 2, 3 e 4 horas após a alta da SRPA, as pacientes recebiam nova visita para reavaliação quanto à presença de dor.

A avaliação da dor pós-operatória foi realizada sem que se soubesse em qual grupo estava incluída a paciente pesquisada.

Considerando-se diferença de 2 pontos na ENV, desvio-padrão de 2,2 pontos e poder de teste de $80 \%$, o tamanho da amostra ficou determinado em no mínimo 20 pacientes por grupo.

Os testes estatísticos utilizados foram o $t$ de Student para dados antropométricos e duração da anestesia e cirurgia, de Friedman para comparar os momentos em cada grupo e de Mann-Whitney para comparar os grupos com relação aos resultados referentes à dor e Qui-quadrado para ocorrência de dor nos períodos de estudo, sendo considerado significativo $p<0,05$.

\section{RESULTADOS}

Os grupos foram homogêneos com relação aos dados antropométricos, à duração da anestesia e cirurgia (Tabela I). 
Tabela I - Dados Antropométricos, Tempo de Duração da Anestesia e da Cirurgia (Média \pm DP)

\begin{tabular}{lccc}
\hline & \multicolumn{2}{c}{ Grupos } & Estatística \\
\cline { 2 - 3 } & \multicolumn{1}{c}{$\mathrm{G} 1$} & $\mathrm{G} 2$ & \\
\hline Idade (anos) & $33,2 \pm 5$ & $34,8 \pm 6$ & $\mathrm{G} 1=\mathrm{G} 2$ \\
Peso (kg) & $64 \pm 9,8$ & $63 \pm 8,7$ & $\mathrm{G} 1=\mathrm{G} 2$ \\
Altura (cm) & $159 \pm 5,9$ & $158,1 \pm 6,3$ & $\mathrm{G} 1=\mathrm{G} 2$ \\
Duração da anestesia (min) & $49,2 \pm 9,5$ & $54 \pm 11$ & $\mathrm{G} 1=\mathrm{G} 2$ \\
Duração da cirurgia (min) & $26,4 \pm 8,4$ & $30,6 \pm 10,4$ & $\mathrm{G} 1=\mathrm{G} 2$ \\
\hline
\end{tabular}

Das 50 pacientes selecionadas para participar do estudo, duas foram excluídas da análise dos resultados porque foram submetidas às duas técnicas de laqueadura. Dentre as 48 pacientes restantes, 25 pertenciam ao grupo 1 e 23 , ao grupo 2.

Vinte e duas pacientes pertencentes ao grupo 1 apresentaram ENV maior que 3 em algum momento do estudo (Tabelas II e III) e necessitaram de analgesia complementar com tramadol (Tabela IV). Já no grupo 2, apenas quatro pacientes apresentaram ENV superior a 3 (Tabelas II e III) e necessitaram de analgesia complementar (Tabela IV).

Tabela II - Mediana, $1^{\circ}$ e $3^{\circ}$ Quartis

\begin{tabular}{lccc}
\hline Momentos & \multicolumn{2}{c}{ Grupos } & \multirow{2}{*}{ Estatística } \\
\cline { 2 - 3 } & $\mathrm{G} 1$ & $\mathrm{G} 2$ & \\
\hline M0 & $0,0[0,0 ; 0,0]$ & $0,0[0,0 ; 0,0]$ & $\mathrm{G} 1=\mathrm{G} 2$ \\
M10 & $1,0[0,0 ; 3,0]$ & $0,0[0,0 ; 0,0]$ & $\mathrm{G} 1>\mathrm{G} 2$ \\
M20 & $1,5[0,0 ; 4,0]$ & $0,0[0,0 ; 0,0]$ & $\mathrm{G} 1>\mathrm{G} 2$ \\
M30 & $2,0[1,0 ; 5,0]$ & $0,0[0,0 ; 0,0]$ & $\mathrm{G} 1>\mathrm{G} 2$ \\
M40 & $3,0[2,0 ; 5,0]$ & $0,0[0,0 ; 0,0]$ & $\mathrm{G} 1>\mathrm{G} 2$ \\
M50 & $3,0[2,0 ; 5,0]$ & $0,0[0,0 ; 0,0]$ & $\mathrm{G} 1>\mathrm{G} 2$ \\
M60 & $3,0[2,0 ; 4,0]$ & $0,0[0,0 ; 0,0]$ & $\mathrm{G} 1>\mathrm{G} 2$ \\
& $\mathrm{M} 0<\mathrm{M} 1=\mathrm{M} 2=\mathrm{M} 3=$ & $\mathrm{M} 0=\mathrm{M} 1=\mathrm{M} 2=\mathrm{M} 3=$ & \\
Estatística & $\mathrm{M} 4=\mathrm{M} 5=\mathrm{M} 6$ & $\mathrm{M} 4=\mathrm{M} 5=\mathrm{M} 6$ & \\
\hline
\end{tabular}

Entre colchetes, referente à escala numérica verbal da dor na SRPA, segundo momento e grupo

Tabela III - Mediana, $1^{\circ}$ e $3^{\circ}$ Quartis

\begin{tabular}{|c|c|c|c|}
\hline \multirow[t]{2}{*}{ Horas } & \multicolumn{2}{|c|}{ Grupos } & \multirow[t]{2}{*}{ Estatística } \\
\hline & G1 & G2 & \\
\hline 1 & $3,5[2,0 ; 4,0]$ & $0,0[0,0 ; 0,0]$ & $\mathrm{G} 1>\mathrm{G} 2$ \\
\hline 2 & $2,0[1,0 ; 3,0]$ & $0,0[0,0 ; 0,0]$ & $\mathrm{G} 1>\mathrm{G} 2$ \\
\hline 3 & $1,0[0,0 ; 3,0]$ & $0,0[0,0 ; 0,0]$ & $\mathrm{G} 1>\mathrm{G} 2$ \\
\hline 4 & $0,0[0,0 ; 2,0]$ & $0,0[0,0 ; 0,0]$ & $\mathrm{G} 1>\mathrm{G} 2$ \\
\hline Estatística & $\mathrm{M} 1>\mathrm{M} 2>\mathrm{M} 3>\mathrm{M} 4$ & $\mathrm{M} 1=\mathrm{M} 2=\mathrm{M} 3=\mathrm{M} 4$ & \\
\hline
\end{tabular}

Entre colchetes, referente à escala numérica verbal da dor na enfermaria, segundo momento e grupo
Tabela IV - Número de Pacientes que Necessitaram de Analgesia Complementar na SRPA e/ou Enfermaria

\begin{tabular}{lcc}
\hline & \multicolumn{2}{c}{ Grupos } \\
\cline { 2 - 3 } & $\mathrm{G} 1(\mathrm{n}=25)$ & $\mathrm{G} 2(\mathrm{n}=23)$ \\
\hline SRPA & 14 & 2 \\
SRPA e enfermaria & 2 & 1 \\
Enfermaria & 6 & 1 \\
Total de pacientes tratadas & $22 *$ & 4 \\
\hline
\end{tabular}

* $p<0,05$

\section{DISCUSSÃo}

Os resultados desta pesquisa demonstraram que a $\mathrm{N}$ - butilescopolamina e a dipirona sódica associadas ao cetoprofeno não foram eficientes na prevenção da dor pós-operatória de pacientes submetidas à laqueadura por laparoscopia pela técnica de pinçamento das tubas uterinas com anéis.

Ador no pós-operatório de laparoscopia ginecológica é significativamente maior após a laqueadura quando comparada à de procedimentos diagnósticos ${ }^{15,16}$ e mais intensa nas primeiras 4 a 6 horas de $\mathrm{PO}^{9}$.

É descrito que a dor, após esterilização laparoscópica, possui intensidade variável e está relacionada à técnica empregada. Os estudos publicados na literatura não apresentam concordância com relação à técnica que daria origem a dores mais intensas. Alguns autores postulam ser aquela que utiliza anéis e pinças ${ }^{5,17}$, enquanto que outros observaram dor mais intensa com diatermia ${ }^{18}$.

A inervação das tubas uterinas está quase que inteiramente sob controle dos nervos autonômicos ${ }^{19}$. A estimulação simpática colinérgica atua via receptores muscarínicos intramurais, aumentando o tônus do músculo liso. Em geral, o espasmo do músculo liso está associado à dor em cólica. A dor que segue a laqueadura laparoscópica tem esta característica, por isso um agente anti-espasmódico seria a opção ideal para o seu tratamento.

O glicopirroplato, agente anticolinérgico, mostrou-se eficaz em reduzir a dor $\mathrm{PO}$, quando administrado antes da cirurgia de pacientes que ligaram as tubas uterinas com clipes de Filshie $^{20}$.

A N-butilescopolamina, antagonista colinérgico muscarínico, é muito utilizada no tratamento da dor em cólicas e tem como vantagem o rápido início de ação quando administrada pela via venosa. Sua ação é predominantemente no gânglio parassimpático intramural do músculo liso abdominal e pélvico $^{21}$. Poderia, portanto, ser utilizada no PO de laqueadura. Contudo, mostrou que é ineficiente na prevenção da dor após esterilização com pinça de Filshie, quando administrada antes ${ }^{13}$ ou após a cirurgia ${ }^{14}$.

Sabe-se que a dor pélvica após a manipulação das tubas, está relacionada à liberação de prostaglandinas. A prostaglandina $\mathrm{PFG}_{2} \alpha$ é encontrada no oviduto humano em concentrações 10 vezes superiores às existentes no plasma. $A$ $\mathrm{PFG}_{2} \alpha$ é encontrada principalmente no istmo, e correlacio- 
na-se com a motilidade desta região, e a $\mathrm{PGE}_{1}$, na ampola. $\mathrm{A}$ manipulação das tubas uterinas libera prostaglandinas, aumentando a freqüência dos impulsos nociceptivos e causando dor ${ }^{12}$. Assim, a dor pode ser tratada com inibidores da síntese de prostaglandinas. Contudo, este grupo de drogas mostrou-se insuficiente para abolir a dor quando foi utilizado como agente único ${ }^{12}$.

O estímulo doloroso periférico e, conseqüentemente, a sensibilização central, ocorre pela presença de substâncias inflamatórias (fator de necrose tumoral-TNF $\alpha$, fator de crescimento tumoral TNG, dentre outras) que facilitam a atividade nos nociceptores do aferente primário. A liberação pré-sináptica da substância $P$ e do glutamato ativam os receptores $\mathrm{N}$-metil-D-aspartato (NMDA) e os do ácido $\alpha$ animo-3 hidroxi-5 metil-4 isoxazol propiônico (AMPA), assim como inibem os receptores do ácido gama amino butírico (GABA), resultando na liberação intracelular de cálcio ${ }^{22}$. Desse modo, aumenta o recrutamento dos neurônios de segunda ordem da condução da dor estimulados por um neurônio de primeira ordem (fibra C). Quando se realiza analgesia, antes de o estímulo nocivo estar presente, este influxo e a dor são menos intensos e a necessidade de analgesia PO, menor ${ }^{23}$.

A ação analgésica preemptiva dos antiinflamatórios não-esteroidais (AINE) não está ainda comprovada ${ }^{24-26}$, entretanto os antagonistas do NMDA podem apresentar esta característica ${ }^{26-30}$

O cetoprofeno, administrado antes do início da anestesia, teve por finalidade promover analgesia preemptiva ${ }^{26}$. Este AINE possui ação não apenas na inibição da síntese de prostaglandinas, mas também na modulação direta da atividade do receptor NMDA, por meio da enzima hepática TDO (triptofano 2,3 dioxigenase), aumentando o isômero do ácido cinurênico no sistema nervoso central, já que a atividade receptora do NMDAé influenciada, seletivamente, peloácido cinurênico na medula ${ }^{31,32}$.

Em pesquisa com cetoprofeno (100 mg) administrado, por via venosa, antes da anestesia e após a cirurgia ou somente após, há resultados significativos que mostram que o grupo que recebeu o cetoprofeno antes do procedimento cirúrgico necessitou de menos analgesia no período entre 12 e 36 horas de $\mathrm{PO}^{26}$.

Quando administrado por via venosa, o cetoprofeno atinge seu pico de concentração plasmática em torno de 4 minutose sua meia-vida de eliminação é de 2 horas, possuindo atividade antitérmica e analgésica ${ }^{24}$. Por estas características farmacológicas e por sua disponibilidade para ser utilizado pela via venosa, trata-se de AINE bem indicado para procedimentos laparoscópicos.

A presente pesquisa, que diferiu das anteriores porque utilizou a N-butilescopolamina e dipirona sódica associadas ao cetoprofeno para analgesia PO de laqueadura laparoscópica, mostrou que esta associação de drogas promoveu analgesia satisfatória apenas nas pacientes que se submeteram à laqueadura pela técnica de diatermia, talvez pelo fato de que esta técnica desencadeie dor de menor intensidade.
Quando se utilizam anéis, a dor é secundária ao estrangulamento das tubas uterinas, levando a isquemia e espasmos ${ }^{5}$, e torna-se mais intensa nas primeiras horas do período $\mathrm{PO}$, uma vez que fatores desencadeantes da dor se mantêm por tempo prolongado ${ }^{17}$.

Para concluir, a associação da N- butilescopolamina e dipirona sódica com o cetoprofeno mostrou que é alternativa de analgesia $\mathrm{PO}$, quando a laqueadura é realizada com a técnica de diatermia.

\section{Efficacy of $\mathbf{N}$-Butylscopolamine and Sodium Dipirone Associated to Ketoprofen for Postoperative Pain Relief of Patients Submitted to Two Different Laparoscopic Sterilization Techniques}

Eliana Marisa Ganem, TSA, M.D.; Isabel C. F. Salem; Fernanda B. Fukushima, M.D.; Giane Nakamura, M.D.; Rogério Dias, M.D.; André L. Fontana, M.D.; Nilton J. Leite, M.D.

\section{INTRODUCTION}

Postoperative abdominal pain is a common complication after laparoscopic procedures ${ }^{1-4}$. Several factors contribute to its onset, such as blood vessels laceration, traumatic nerves traction, inflammatory mediators release associated to rapid peritoneal distention, and phrenic nerve stimulation by residual $\mathrm{CO}_{2}$ in the abdominal cavity leading to referred shoulder pain ${ }^{5}$.

Laparoscopic sterilization is one of the most painful gynecologic laparoscopic procedures. PO pain severity varies with the technique of the procedure.

Post-sterilization pain may be treated with local anesthetics instilled in uterine tubes and mesosalpinx, with NSAIDs and opioids ${ }^{6-11}$. Anti-inflammatory drugs could not abolish pain when used as single agents ${ }^{12}$.

Patients submitted to sterilization refer colic pain in the immediate $\mathrm{PO}$ period and $\mathrm{N}$-butylscopolamine and sodium dipirone (Buscopan Composto ${ }^{\circledR}$ ), for their anti-spasmodic and analgesic properties, associated to ketoprofen, could be an option to prevent PO pain. There are, however, studies questioning the efficacy of $\mathrm{N}$-butylscopolamine to treat $\mathrm{PO}$ pain after sterilization with Filshie's clamps ${ }^{13,14}$.

Since there is no report in the literature with $\mathrm{N}$-butylscopolamine and sodium dipirone plus ketoprofen to control PO pain after laparoscopic sterilization, we have studied the efficacy of this association in patients submitted to two different surgical techniques, that is, diathermy and tubal rings. 


\section{METHODS}

After the Clinical Research Ethics Committee approval and their written consent, participated in this study 50 female patients, aged 23 to 47 years, physical status ASA I and II and body mass index below 30 , who were submitted to laparoscopic sterilization. Exclusion criteria were patients under anti-inflammatory or analgesic drugs in the preoperative period, alcohol and drug users and those who were menstruated. Patients were randomly distributed in two groups according to the surgical technique: G1 - tubal occlusion with rings, and G1, tubal occlusion with diathermy. All patients received intravenous $\mathrm{N}$-butylscopolamine $(20 \mathrm{mg})$ and sodium dipirone $(2500 \mathrm{mg})$ plus ketoprofen $(100 \mathrm{mg})$ immediately before anesthetic induction.

After an 8-hour fasting period, patients were premedicated with $7.5 \mathrm{mg}$ oral midazolam, 60 minutes before being referred to the operating center. In the operating room, after obtaining venous access, lactated Ringer's solution was installed (10 $\mathrm{mL} . \mathrm{kg}^{-1}$ ) and ondansetron (4 mg) was administered to prevent nausea and vomiting.

Monitoring consisted of ECG at $D_{\|}$lead, sphygmomanometer and pulse oximetry. Anesthesia was induced with sufentanil $\left(0.5 \mu \mathrm{g} . \mathrm{kg}^{-1}\right)$ and propofol $\left(2 \mathrm{mg} \cdot \mathrm{kg}^{-1}\right)$. Atracurium $\left(0.5 \mathrm{mg} \cdot \mathrm{kg}^{-1}\right)$ was administered to help tracheal intubation. After manual ventilation with $100 \%$ oxygen $\left(\mathrm{O}_{2}\right)$, tracheal intubation, capnography installation and orogastric tube insertion to relief air built up in the stomach during manual ventilation were achieved and isoflurane administration was started. Rebreathing system with nitrous oxide $\left(\mathrm{N}_{2} \mathrm{O}\right)$ in 0.4 oxygen inspired fraction was the technique of choice. Tidal volume was adjusted to $10 \mathrm{~mL} \cdot \mathrm{kg}^{-1}$ and respiratory rate was adjusted to maintain end tidal $\mathrm{CO}_{2}\left(\mathrm{P}_{\mathrm{ET}} \mathrm{CO}_{2}\right)$ in approximately $30 \mathrm{mmHg}$. At the end of anesthesia and after neuromuscular block reversion with intravenous atropine $(1 \mathrm{mg})$ and neostigmine $(1.5 \mathrm{mg})$ patients were extubated as soon as they recovered enough spontaneous ventilation and consciousness, when they were referred to the PACU. At PACU patients were asked about pain every 10 minutes for one hour. Pain was evaluated by the verbal numeric scale (VNS) varying from 0 to 10 , being 0 no pain and 10 the worst possible pain. When pain severity was beyond 3 , patients were given intravenous tramadol (50 $\mathrm{mg})$. When pain persisted, tramadol was repeated $(50 \mathrm{mg})$. If it still persists, intravenous morphine was administered.

In the ward, patients were evaluated 1, 2, 3 and 4 hours after PACU discharge to re-evaluate the presence of pain.

$\mathrm{PO}$ pain was evaluated without knowing the group to which the patient belonged.

Considering 2 points difference in VNS, 2.2 points standard deviation and test power of $80 \%$, sample size was determined as at least 20 patients per group.

Student's $t$ test was used for demographics data, surgery and anesthesia duration, Friedman's test was used to compare moments within each group and Mann-Whitney test was used to compare groups to pain results. Chi-square test was used to analyze pain during the study periods, being considered significant $p<0.05$.

\section{RESULTS}

Groups were homogeneous in demographics data, anesthesia and surgery duration (Table I).

Table I - Demographics Data, Anesthesia and Surgery Duration (Mean \pm SD)

\begin{tabular}{lccc}
\hline & \multicolumn{2}{c}{ Groups } & \multirow{2}{*}{ Statistics } \\
\cline { 2 - 3 } & \multicolumn{1}{c}{$\mathrm{G} 1$} & $\mathrm{G} 2$ & \\
\hline Age (years) & $33.2 \pm 5$ & $34.8 \pm 6$ & $\mathrm{G} 1=\mathrm{G} 2$ \\
Weight (kg) & $64 \pm 9.8$ & $63 \pm 8.7$ & $\mathrm{G} 1=\mathrm{G} 2$ \\
Height (cm) & $159 \pm 5.9$ & $158.1 \pm 6.3$ & $\mathrm{G} 1=\mathrm{G} 2$ \\
Anesthesia duration (min) & $49.2 \pm 9.5$ & $54 \pm 11$ & $\mathrm{G} 1=\mathrm{G} 2$ \\
Surgery duration (min) & $26.4 \pm 8.4$ & $30.6 \pm 10.4$ & $\mathrm{G} 1=\mathrm{G} 2$ \\
\hline
\end{tabular}

From 50 patients selected for the study, 2 were excluded from the analysis because they were submitted to both sterilization techniques. Among 48 remaining patients, 25 belonged to group 1 and 23 to group 2.

Twenty-two group 1 patients presented VNS above 3 at some point of the study (Tables II and III) and needed additional analgesia with tramadol (Table IV). In group 2, only 4 patients presented VNS above 3 (Tables II and III) and needed additional analgesia (Table IV).

Table II - Median, $1^{\text {st }}$ and $3^{\text {rd }}$ Quartiles

\begin{tabular}{lccc}
\hline Moments & \multicolumn{2}{c}{ Groups } & \multirow{2}{*}{ Statistics } \\
\cline { 2 - 3 } & $\mathrm{G} 1$ & $\mathrm{G} 2$ & \\
\hline M0 & $0.0[0.0 ; 0.0]$ & $0.0[0.0 ; 0.0]$ & $\mathrm{G} 1=\mathrm{G} 2$ \\
M10 & $1.0[0.0 ; 3.0]$ & $0.0[0.0 ; 0.0]$ & $\mathrm{G} 1>\mathrm{G} 2$ \\
M20 & $1.5[0.0 ; 4.0]$ & $0.0[0.0 ; 0.0]$ & $\mathrm{G} 1>\mathrm{G} 2$ \\
M30 & $2.0[1.0 ; 5.0]$ & $0.0[0.0 ; 0.0]$ & $\mathrm{G} 1>\mathrm{G} 2$ \\
M40 & $3.0[2.0 ; 5.0]$ & $0.0[0.0 ; 0.0]$ & $\mathrm{G} 1>\mathrm{G} 2$ \\
M50 & $3.0[2.0 ; 5.0]$ & $0.0[0.0 ; 0.0]$ & $\mathrm{G} 1>\mathrm{G} 2$ \\
M60 & $3.0[2.0 ; 4.0]$ & $0.0[0.0 ; 0.0]$ & $\mathrm{G} 1>\mathrm{G} 2$ \\
& $\mathrm{M} 0<\mathrm{M} 1=\mathrm{M} 2=\mathrm{M} 3=$ & $\mathrm{M} 0=\mathrm{M} 1=\mathrm{M} 2=\mathrm{M} 3=$ \\
Statistics & $\mathrm{M} 4=\mathrm{M} 5=\mathrm{M} 6$ & $\mathrm{M} 4=\mathrm{M} 5=\mathrm{M} 6$ & \\
\hline
\end{tabular}

In brackets, related to pain verbal numeric scale in the PACU, according to moment and group

Table III - Median, $1^{\text {st }}$ and $3^{\text {rd }}$ Quartiles

\begin{tabular}{lccl}
\hline Hours & \multicolumn{2}{c}{ Groups } & \multirow{2}{*}{ Statistics } \\
\cline { 2 - 3 } & $\mathrm{G} 1$ & $\mathrm{G} 2$ & \\
\hline 1 & $3.5[2.0 ; 4.0]$ & $0.0[0.0 ; 0.0]$ & $\mathrm{G} 1>\mathrm{G} 2$ \\
2 & $2.0[1.0 ; 3.0]$ & $0.0[0.0 ; 0.0]$ & $\mathrm{G} 1>\mathrm{G} 2$ \\
3 & $1.0[0.0 ; 3.0]$ & $0.0[0.0 ; 0.0]$ & $\mathrm{G} 1>\mathrm{G} 2$ \\
4 & $0.0[0.0 ; 2.0]$ & $0.0[0.0 ; 0.0]$ & $\mathrm{G} 1>\mathrm{G} 2$ \\
Statistics & $\mathrm{M} 1>\mathrm{M} 2>\mathrm{M} 3>\mathrm{M} 4$ & $\mathrm{M} 1=\mathrm{M} 2=\mathrm{M} 3=\mathrm{M} 4$ & \\
\hline
\end{tabular}

In brackets, related to pain verbal numeric scale in the ward, according to moment and group 
Table IV - Number of Patients Needing Additional Analgesia in the PACU and/or Ward

\begin{tabular}{lcc}
\hline & \multicolumn{2}{c}{ Groups } \\
\cline { 2 - 3 } & $\mathrm{G} 1(\mathrm{n}=25)$ & $\mathrm{G} 2(\mathrm{n}=23)$ \\
\hline PACU & 14 & 2 \\
PACU and/or ward & 2 & 1 \\
Ward & 6 & 1 \\
Total treated patients & $22{ }^{*}$ & 4 \\
\hline
\end{tabular}

${ }^{*} p<0.05$

\section{DISCUSSION}

Our results have shown that N-butylscopolamine and sodium dipirone associated to ketoprofen were ineffective to prevent $\mathrm{PO}$ pain in patients submitted to laparoscopic sterilization with tubal rings.

Post-gynecologic laparoscopy pain is significantly worse after sterilization as compared to diagnostic procedures ${ }^{15,16}$, and is more severe in the first 4 to 6 PO hours ${ }^{9}$.

It has been described that pain after laparoscopic sterilization is variable and related to the technique of the procedure. Studies in the literature do not agree with the technique that would originate most severe pain. Some authors state that it is the technique using rings and clamps ${ }^{5,17}$, while others have observed more severe pain with diathermy ${ }^{18}$.

Tubal innervation is almost totally under autonomic nerve control $^{19}$. Cholinergic sympathetic stimulation acts via intramural muscarinic receptors, increasing smooth muscle tone. In general, smooth muscle spasm is associated to colic pain. Pain following laparoscopic sterilization has this characteristic, so an anti-spasmodic agent would be ideal for its treatment. Glycopyrrolate an anticholinergic drug was efficient to decrease $P O$ pain when administered before surgery in patients submitted to tubes occlusion by Filshie ${ }^{15}$ clamp technique ${ }^{20}$. The N-butylscopolamine, a muscarinic cholinergic antagonist, is widely used to treat colic pain with the advantage of fast onset when intravenously administered. It acts predominantly on the intramural parasympathetic ganglion of pelvic and abdominal smooth muscles ${ }^{21}$. So, it could be used for sterilization PO period. However, it has been shown ineffective to prevent pain after sterilization with Filshie's clamp, whether administered before ${ }^{13}$ or after surgery ${ }^{14}$.

It is known that pelvic pain after tubal manipulation is related to prostaglandin release. $P F G_{2} \alpha$ prostaglandin is found in human oviduct in concentrations 10 times higher than plasma concentrations. $\mathrm{PFG}_{2} \alpha$ is mainly found in the isthmus and is correlated to mobility of this region, and $P \mathrm{PE}_{1}$ is found in the ampoule. Tubal manipulation releases prostaglandins increasing the frequency of nociceptive impulses and causing pain ${ }^{12}$. So, pain may be treated with prostaglandin synthesis inhibitors. However, this group of drugs was ineffective in abolishing pain when used as single agent ${ }^{12}$.

Peripheral pain stimulation and, consequently, central sensitization, are caused by the presence of inflammatory sub- stances (tumor necrosis factor-TNF $\alpha$, tumor growth factor, among others) which help nociceptors activity on primary afferent. Pre-synaptic $P$ substance and glutamate release activates $\mathrm{N}$-methyl-aspartate (NMDA) and $\alpha$ amino- 3 hydroxi-5 methyl-4 isoxazol propionic acid (AMPA) receptors, as well as inhibits gama amino butyric acid receptors (GABA), resulting in intracellular calcium release ${ }^{22}$. So, the recruitment of second order pain conduction neurons stimulated by a first order neuron ( $\mathrm{C}$ fiber) is increased. When the analgesic is administered before the presence of noxious stimulation, in experimental conditions, this inflow and pain are less severe with lower need for PO analgesia ${ }^{23}$.

Preemptive analgesic action of non-steroidal anti-inflammatory drugs (NSAID) is still not proven ${ }^{24-26}$, however NMDAantagonists may have this characteristic ${ }^{26-30}$. Ketoprofen given before anesthesia aimed at promoting preemptive analgesia ${ }^{26}$. This NSAID acts not only by inhibiting prostaglandin synthesis, but also by directly modulating NMDA receptor activity through the liver enzyme TDO (triptophane 2,3 dioxygenase), increasing cinurenic acid isomer in the central nervous system, and NMDA receptor activity is selectively influenced by spinal cord cinurenic acid ${ }^{31,32}$.

A study with intravenous ketoprofen $(100 \mathrm{mg})$ before anesthesia and after surgery, or just after surgery, has presented significant results showing that the group receiving ketoprofen before surgery needed less analgesia from 12 to 36 PO hours ${ }^{26}$.

Intravenous ketoprofen reaches its plasma concentration peak in approximately 4 minutes and its excretion half-life is 2 hours, having anti-thermal and analgesic activities ${ }^{24}$. For those pharmacologic characteristics and its availability for intravenous administration it is an adequate indication for laparoscopic procedures.

Our study, which was different from previous studies because we have used $\mathrm{N}$-butylscopolamine and sodium dipirone associated to ketoprofen for post laparoscopic sterilization analgesia, has shown that this association was only able to promote satisfactory analgesia in patients submitted to diathermy, maybe because this technique triggers less severe pain. When rings are used, pain is secondary to tubal strangulation, leading to ischemia and spasms ${ }^{5}$, and becomes more severe in the first $P O$ hours, since pain triggering factors are maintained for a long time ${ }^{17}$.

In conclusion, $\mathrm{N}$-butylscopolamine and sodium dipirone associated to ketoprofen are a PO analgesic alternative for sterilization with diathermy technique.

\section{REFERÊNCIAS - REFERENCES}

01. Skacel M, Sengupta P, Plantevin OM - Morbidity after day case laparoscopy. A comparison of two techniques of tracheal anaesthesia. Anaesthesia, 1986;41:537-541.

02. Collins KM, Plantevin OM, Docherty PW - Comparison of atracurium and alcuronium in day- case gynaecological surgery. Anaesthesia, 1984;39:1130-1134. 
03. Ganem EM, Castiglia YMM, Módolo NSP et al - Laparoscopia ginecológica: estudo retrospectivo de complicações intra e pós-operatórias. Rev Bras Anestesiol, 1995;45:165-172.

04. Collins KM, Docherty PW, Plantevin OM - Postoperative morbidity following gynaecological outpatient laparoscopy. A reappraisal of the service. Anaesthesia, 1984;39:819-822.

05. Dobbs FF, Kumar V, Alexander JI et al - Pain after laparoscopy related to posture and ring versus clip sterilization. $\mathrm{Br} \mathrm{J}$ Obstet Gynaecol, 1987;94:262-266.

06. Tool Al, Kammerer-Doak DN, Nguyen CM et al - Postoperative pain relief following laparoscopic tubal sterilization with silastic bands. Obstet Gynecol, 1997;90:731-734

07. Budd J, Whyte J, Wilson M et al - A comparison of tramadol with morphine for postoperative analgesia following day case laparoscopic sterilization. Eur J Anaesthesiol, 2000;17:A34.

08. Wheatley SA, Millar JM, Jadad AR - Reduction of pain after laparoscopic sterilisation with local bupivacaine; a randomised, parellel, double-blind trial. Br J Obstet Gynaecol, 1994;101: 443-446.

09. Buggy DJ, Wall C, Carton EG - Preoperative or postoperative diclofenac for laparoscopic tubal ligation. $\mathrm{Br} \mathrm{J}$ Anaesth, 1994;73:767-770.

10. Chui PT, Gin T - A comparison between ketorolac and diclofenac in laparoscopic sterelization. Eur J Anaesthesiol, 1995;12: 597-601.

11. Putland AJ, McCluskey A - The analgesic efficacy of tramadol verus ketorolac in day-case laparoscopic sterilisation. Anaesthesia, 1999;54:382-385.

12. Alexander $\mathrm{JI}$ - Pain after laparoscopy. $\mathrm{Br} \mathrm{J}$ Anaesth, 1997;79:369-378.

13. Wilson CM, Lillywhite N, Matta B et al - Intravenous buscopan for analgesia following laparoscopic sterilisation. Anaesthesia, 1999;54:389-392.

14. Habib A, Anderson S, Francis $S$ et al - Buscopan for the treatment of pain after laparoscopic sterilisation. Anaesthesia, 2001;56:174-176.

15. Edwards ND, Barclay K, Catling SJ et al - Day case laparoscopy: a survey of postoperative pain and an assessment of the value of diclofenac. Anaesthesia, 1991;46:1077-1080.

16. Davis A, Millar JM - Postoperative pain: a comparison of laparoscopic sterilisation and diagnostic laparoscopy. Anaesthesia, 1988;43:796-797.

17. Chi IC, Cole LP - Incidence of pain among women undergoing laparoscopic sterilization by electrocoagulation, the spring-loaded clip, and the tubal ring. Am J Obstet Gynecol, 1979;135:397-401.

18. Lawson S, Cole RA, Templeton AA - The effect of laparoscopic sterilisation by diathermy or silastic bands on post-operative pain, menstrual symptoms and sexuality. Br J Obstet Gynaecol, 1979;86:659-663.

19. Smith BE, MacPherson GH, de Jonge $M$ et al - Rectus sheath and mesosalpinx block for laparoscopic sterilization. Anaesthesia, 1991;46:875-877.

20. Guard BC, Wiltshire SJ - The effect of glycopyrrolate on postoperative pain and analgesic requirements following laparoscopic sterilisation. Anaesthesia, 1996;51:1173-1175.

21. Hupscher DN, Dommerholt O - Action and side effects of small doses of buscopan in gastroduodenal radiography. A prospective study in 300 patients. Diagn Imaging Clin Med, 1984:53:77-86

22. Finnerup NB, Sindrup SH, Jensen TS - Anticonvulsant analgesics in peripheral and central neuropathic pain. Pain Med Pal Care, 2004,3:42-52

Revista Brasileira de Anestesiologia

Vol. 55, № 4, Julho - Agosto, 2005
23. Woolf CJ, Chong MS - Preemptive analgesia-treating postoperative pain by preventing the establishment of central sensitization. Anesth Analg, 1993;77:362-379.

24. Likar R, Krumpholz R, Pipam W - Randomized, double-blind study with ketoprofen in gynecologic patients. Preemptive analgesia study following the Brevik-Stubhaug design. Anaesthesist, 1998;47:303-310.

25. Likar R, Krumpholz R, Mathiaschitz K et al - The preemptive action of ketoprofen. Randomized, double-blind study with gynecologic operations. Anaesthesist, 1997;46:186-190.

26. Wnek W, Zajaczkowska R, Wordliczek J et al - Influence of pre-operative ketoprofen administration (preemptive analgesia) on analgesic requirement and the level of prostaglandins in the early postoperative period. Pol J Pharmacol, 2004;56: 547-552.

27. Wu C, Yu J, Yeh C et al - Preincisional dextromethorphan treatment decreases postoperative pain and opioid requirement after laparoscopic cholecystectomy. Anesth Analg, 1999;88: 1331-1334.

28. Helmy SA, Bali A - The effect of the preemptive use of the NMDA receptor antagonist dextromethorphan on postoperative analgesic requirements. Anesth Analg, 2001;92:739-744.

29. Fu ES, Miguel R, Scharf JE - Preemptive ketamine decreases postoperative narcotic requirements in patients undergoing abdominal surgery. Anesth Analg, 1997;84;1086-1090.

30. Suto Y, Yamaguchi H, Sato S et al - Preoperative Ketamine augments postoperative pain relief by epidural morphine in patients undergoing gynecological surgeries. Anesth Analg, 1998;86:(Suppl2): 319S.

31. Birch PJ, Grossman CJ, Hayes AG - Kynurenic acid antagonises responses to NMDA via an action at the strychnine-insensitive glycine receptor. Eur J Pharmacol, 1988;154:85-87.

32. McCormack K - The spinal actions of nonsteroidal anti-inflammatory drugs and the dissociation between their anti-inflammatory and analgesic effects. Drugs, 1994;47:(Suppl5):28-47.

\section{RESUMEN}

Ganem EM, Salem ICF, Fukushima FB, Nakamura G, Dias R, Fontana AL, Leite NJ - Eficacia de la N-Butilescopolamina y Dipirona Sódica Asociadas al Cetoprofeno en el Alivio del Dolor Pos-Operatorio de Pacientes Sometidas a Dos Técnicas Diferentes de Laqueadura por Laparoscopia

JUSTIFICATIVA Y OBJETIVOS: La laqueadura laparoscópica $(L L)$ es uno de los procedimientos más dolorosos y la intensidad del dolor varia con la técnica seleccionada, siendo más intensa con la técnica de oclusión de las trompas uterinas con anillo. Las pacientes sometidas a la $L L$ refieren dolor en cólico en el período $\mathrm{PO}$ y la N-butilescopolamina y la dipirona sódica, por sus propiedades anti-espasmódicas y analgésicas, asociadas a las propiedades antiinflamatorias del cetoprofeno, pueden ser opción para la prevención y tratamiento del dolor. No existen, en la literatura, trabajos que comprueben la eficiencia de la N-butilescopolamina y de la dipirona sódica en el tratamiento del dolor PO de laqueadura laparoscópica. El objetivo de ésta fue estudiar la eficacia de la $N$-butilescopolamina y de la dipirona sódica asociadas al cetoprofeno, en la prevención del dolor $P O$ en pacientes sometidas a la LL, con dos técnicas diferentes - diatermia y pinzamiento con anillo.

MÉTODO: Participaron del estudio 50 pacientes, estado físico ASA I y II, con edad entre 23 y 47 años. Las pacientes fueron distribuidas eventualmente en dos grupos: $G 1$ - oclusión de las trompas uterinas con anillos, G2 - oclusión de las trompas 
uterinas con diatermia. Todas las pacientes recibieron $\mathrm{N}$-butilescopolamina $(20 \mathrm{mg})$ y dipirona sódica $(2500 \mathrm{mg})$ y cetoprofeno (100 mg), por vía venosa, inmediatamente antes de la inducción de la anestesia. El dolor fue evaluado por el criterio de escala numérica verbal, variando de 0 a 10 , siendo 0 ausencia de dolor y 10 el máximo de dolor, a cada 10 minutos en la primera hora, en la sala de recuperación pos-anestésica (SRPA) y en la $1^{a}, 2^{a}, 3^{a}$ y $4^{a}$ horas después del alta de la SRPA. Dolor con intensidad mayor que 3 era tratada con tramadol (100 $\mathrm{mg}$ ), por vía venosa. La evaluación de la $D$ fue realizada sin que se supiese a que grupo pertenecía la paciente. Para análisis estadístico, prueba $t$ de Student, Mann-Whitney y Friedman.
RESULTADOS: Ambos grupos fueron idénticos con relación a la edad, al peso, a la altura, duración de la cirugía y anestesia. Las pacientes de G1 presentaron mayores resultados de dolor que las de G2, en todos los momentos del estudio. Valores estadísticamente significativos: $80 \%$ de las pacientes de $\mathrm{G} 1$ y $16 \%$ de G2 necesitaron tramadol en algún momento del estudio.

CONCLUSIONES: La N-butilescopolamina y la dipirona sódica asociadas al cetoprofeno mostraron que es una alternativa de analgesia pos-operatoria cuando la laqueadura es realizada con la técnica de diatermia. 